\title{
Avaliação do controle externo da qualidade nos laboratórios clínicos do Rio de Janeiro de 2006 a 2008
}

Primeira submissão em 26/12/09 Última submissão em 20/05/10 Aceito para publicação em 20/05/10 Publicado em 20/10/10

\section{Evaluation of external quality assessment in clinical laboratories from Rio de Janeiro in the years 2006-2008}

Josefa Sieira Caamaño Chaves'; Victor Augustus Marin²

\section{unitermos \\ Controle externo da qualidade}

Ensaios de proficiência

Laboratórios clínicos

\section{resumo}

Introdução: O Controle Externo da Qualidade (CEQ) é uma ferramenta importante para a garantia da qualidade das análises laboratoriais, auxiliando o laboratório a avaliar a eficiência da fase analítica de seus processos. Um resultado errôneo de uma análise prejudica a conclusão do diagnóstico de uma enfermidade e a indicação correta do tratamento a ser tomado. As inspeções sanitárias em laboratórios clínicos (LCS) no estado do Rio de Janeiro são feitas com base no roteiro de inspeção sanitária publicado pela Portaria SES/CVS 743, de junho de 2006, na RDC 302/2005 e legislação vigente. Os resultados observados quanto ao CEQ, do total de 347 inspeções realizadas pelo Setor de Laboratórios de Análises Clínicas da Secretaria de Saúde e Defesa Civil (LAC-SESDEC) no decorrer dos anos pesquisados, são apresentados a seguir: em 2006, entre as 133 inspeções realizadas em Laboratórios de Análises Clínicas, $44 \%$ dos estabelecimentos não possuíam contrato com provedor de ensaios de proficiência (EPs); em 2007, entre as 95 inspeções realizadas em Laboratórios de Análises Clínicas, 38\% não possuíam contrato com provedor de EPs; em 2008, entre as 119 inspeções realizadas em Laboratórios de Análises Clínicas, $35 \%$ não possuíam contrato com provedor de EPs. Dos 214 laboratórios clínicos inspecionados nos anos de 2007 e 2008, 62\% (134 estabelecimentos) possuíam CEQ, entretanto, desses 134 estabelecimentos, $65 \%$ não registravam as ações corretivas relacionadas com as não conformidades detectadas em relatório emitido pelo provedor de EPs. Método: No presente estudo, foi avaliada a distribuição percentual dos resultados das inspeções sanitárias realizadas em LCs no estado do Rio de Janeiro de 2006 a 2008, cadastrados na Secretaria Estadual de Saúde e Defesa Civil (SESDEC), relacionando o número de LCs que não atendem às exigências sanitárias, item: "Controle Externo da Qualidade - CEQ da Portaria 743/2006", e a natureza e a iniciativa desses estabelecimentos: LC intra e extra-hospitalares (hospitais públicos ou privados, próprios ou terceirizados). Objetivo: Avaliação percentual do CEQ em LCs no estado do Rio de Janeiro ao longo do período analisado, com relação à natureza desses estabelecimentos.

\section{abstract}

Introduction: External Quality Control (EQC) is an important tool for quality assurance, assisting the laboratory in evaluating the efficiency of the analytical phase of its processes. An erroneous result of an analysis hinders the conclusion of diagnosis and the suitable treatment prescription to be followed. Health inspections in Clinical Laboratories (CL) in the State of Rio de Janeiro are made based on a sanitary inspection procedure issued by Directive SES/CVS 743, June 2006, RDC 302/2005 and current Brazilian legislation. EQC results observed from the total of 347 inspections conducted by the Division of Clinical Analysis Laboratories of the Department of Health and Civil Defense (LAC-SESDEC) during the period under review are presented below: 44\% of 133 inspections performed in Clinical Analysis Laboratories had no contract with a proficiency testing provider in 2006; 38\% of 95 inspections conducted in Clinical Analysis Laboratories did not have a contract with any proficiency testing provider in 2007; and 35\% of 119 inspections conducted in Clinical Analysis Laboratories had no contract with any proficiency testing provider in 2008. Sixty-two percent (134 laboratories) of $214 \mathrm{CL}$ inspected in the years 2007 and 2008 had EQC, however, $65 \%$ of them did not record the corrective actions related to the nonconformities identified in a report issued by the proficiency testing provider. Method: In this study, we evaluated the percentage distribution of the results of health inspections conducted in CL in the State of Rio de Janeiro from 2006 to 2008, registered at SESDEC, stating the number of CL that did not meet health requirements (item: "External Quality Control - EQC-Directive 743/2006") and the nature and the sector of these laboratories: intra and extra hospital clinical laboratories (publicly or privately owned hospitals and outsourced hospitals). Objective: Statistic evaluation of EQC in CL in the State of Rio de Janeiro during the period under review as to the nature of these laboratories. key words

\section{External quality}

assessment

Proficiency assay

Clinical laboratories

1. Especialista em Vigilância Sanitária; Farmacêutica-Bioquímica - Secretaria de Estado de Saúde e Defesa Civil/Superintendência de Vigilância Sanitária (SESDEC/SUVISA).

2. Pós-doutor em Vigilância Sanitária pelo Instituto Nacional de Controle de Qualidade em Saúde (INCQS); Vice-diretor de Pesquisa, Ensino e Projetos Estratégicos do INCQS/Fundação Oswaldo Cruz (FIOCRUZ). 


\section{Introdução}

Desde os anos 1990, os Laboratórios Clínicos (LCs) vêm sofrendo mudanças significativas em função do desenvolvimento tecnológico, surgimento de novas técnicas de diagnóstico com alta precisão, eficiência e processamento de maiores volumes.

Considerando a necessidade de normalização do funcionamento dos $\mathrm{LCS}$, a proteção do usuário e do meio ambiente e levando em conta a relevância da qualidade dos exames laboratoriais para apoio ao diagnóstico eficaz, em 13 de outubro de 2005 a Agência Nacional de Vigilância Sanitária (ANVISA) publicou a Resolução RDC no 302/2005, que "dispõe sobre Regulamento Técnico para funcionamento de Laboratórios Clínicos no Brasil".

Contemplando este princípio e com base na RDC 302/2005, em junho de 2006, por meio da Portaria SES/ SVS 743, foi publicado o roteiro para inspeção sanitária em Laboratórios Clínicos no Estado do Rio de Janeiro.

É fato que um resultado errôneo de uma análise laboratorial prejudica a conclusão do diagnóstico de uma enfermidade e a indicação correta do tratamento a ser adotado. Thomas ${ }^{(5)}$ cita em seu trabalho que a principal intenção de um esquema de controle externo da qualidade (CEQ) em laboratórios clínicos é apoiar a melhoria da qualidade dos serviços prestados pelos laboratórios participantes em benefício do paciente.

A crescente atenção dada à segurança dos pacientes $\mathrm{e}$ a consciência de que as informações fornecidas pelos LCs causam impactos diretos sobre o tratamento recebido pelos pacientes demonstram que hoje é uma prioridade para os LCs reduzir suas taxas de erro e promover excelente nível de qualidade para os serviços prestados ${ }^{(4)}$.

\section{Objetivos}

Avaliar o percentual dos dados relativos ao CEQ nos LCs, itens 1.6 e 7.2.4 do roteiro de inspeção sanitária da Portaria SES/CVS no 743, de 28 de junho de 2006, gerados pelas inspeções procedidas no período de 2006 a $2008 \mathrm{em}$ LCs no âmbito do estado do Rio de Janeiro e, dessa forma, gerar conhecimentos para discussão e adoção de medidas para melhoria desses serviços.

Estabelecer o perfil das unidades que não possuem CEQ, de forma a orientar a priorização de ações de vigilância sanitária.
Estabelecer o percentual de estabelecimentos que, apesar de possuírem CEQ, não procedem a ações corretivas relacionadas com as não conformidades observadas após disponibilização de relatório por parte de provedor de ensaios de proficiência (EPs).

\section{Métodos e procedimentos}

Os LCs em funcionamento em diferentes municípios do estado do Rio de Janeiro, cadastrados na Superintendência de Vigilância Sanitária (SUVISA), foram avaliados durante as inspeções sanitárias no período de 2006 a 2008. Durante as inspeções, foi preenchido o roteiro de inspeção sanitária da Portaria SES/CVS 743, de junho de 2006. A partir desse roteiro foram coletados dados relativos aos itens 1.6 e 7.2.4 (relacionados ao CEQ) e que se relacionam com os itens 9.3.1 e 9.3.4 da RDC 302/2005.

Cadastrados no setor de Laboratórios de Análises Clínicas (LAC) da Secretaria de Estado de Saúde e Defesa Civil (SESDEC) há 616 estabelecimentos, entre laboratórios clínicos e postos de coleta.

A meta de inspeções anuais no setor de LAC do Departamento de Fiscalização Sanitária em Estabelecimentos de Saúde (DEFES) - SESDEC é de 30\%, em decorrência da complexidade do serviço, do número de estabelecimentos cadastrados e da capacidade operacional do setor, incluindo laboratórios e postos de coleta, sendo que os EPs aplicamse somente a LCs.

As amostras serão relacionadas nos resultados, quanto à sua natureza, em intra-hospitalares - os LCs podem se situar no interior de hospitais públicos (estaduais, municipais ou federais, que poderão, ainda, ser próprios ou terceirizados, situação verificada a partir de 2007 em hospitais públicos estaduais) ou privados, que poderão ser próprios ou terceirizados - ou extra-hospitalares.

A pesquisa foi dividida em duas etapas: a primeira estabeleceu uma planilha de dados fornecidos pelo roteiro de inspeção e a segunda, avaliação estatística dos dados gerados.

\section{Resultados}

Em 2006, o LAC/DEFES/SESDEC inspecionou 133 LCs. Desse montante (Figura 1), 44\% não possuíam contrato com provedor de EP/CEQ. Desses 44\%, 25 unidades de LC situavam-se no interior de hospitais privados (seis terceirizados 


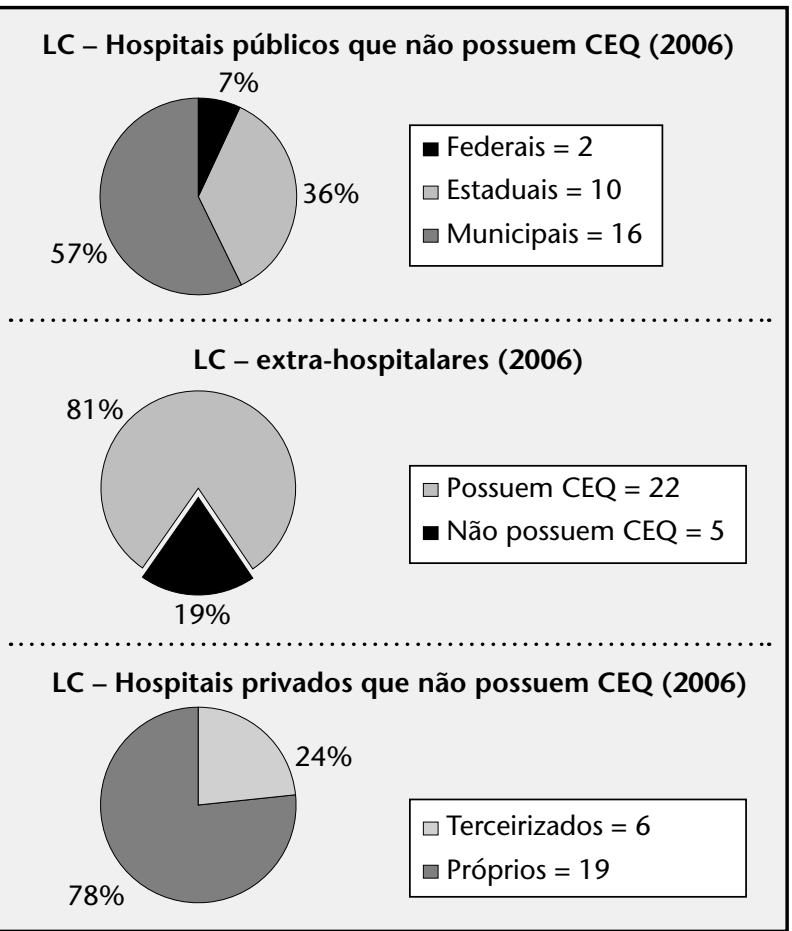

Figura 1 - Total de LCS (ano 2006) que não possuíam CEQ = 58 LC: laboratório clínico; CEQ: controle externo de qualidade.

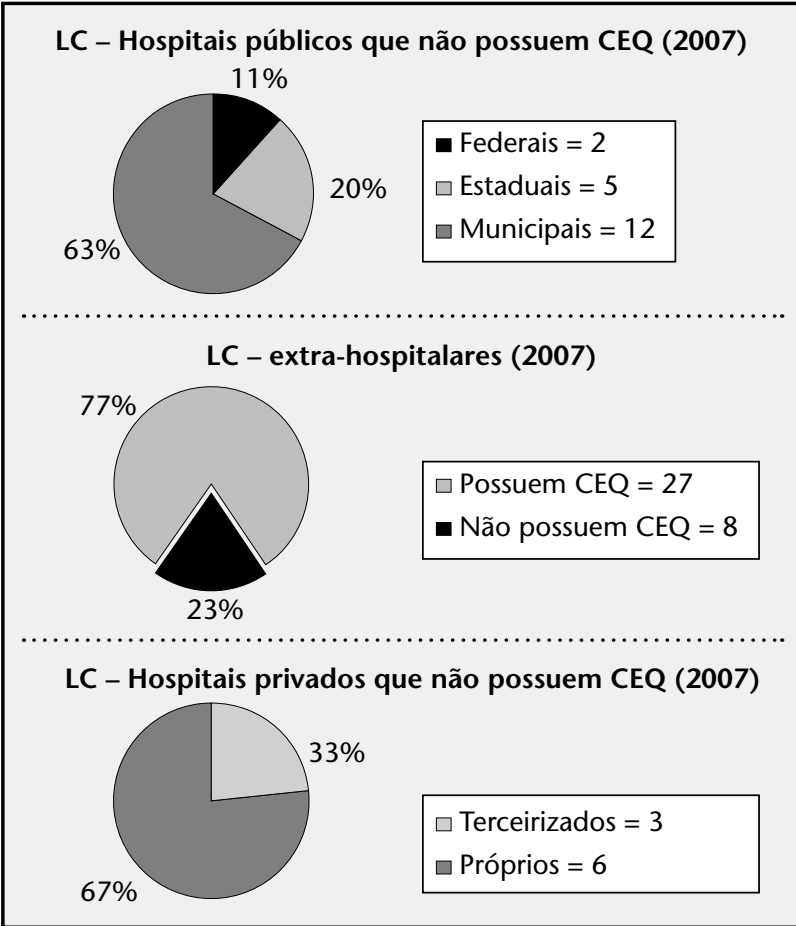

Figura 2 - Total de LCS (ano 2007) que não possuíam CEQ = 36 LC: laboratório clínico; CEQ: controle externo de qualidade.

e 19 próprios); 28 no interior de hospitais públicos (todos próprios) e cinco eram extra-hospitalares.

Em 2007, o LAC/DEFES/SESDEC inspecionou 95 LCs; desses (Figura 2), 38\% não possuíam contrato com provedor de EP/CEQ. O número de inspeções foi consideravelmente menor que em 2006 devido a questões orçamentárias. Dos $40 \%$, nove unidades de LC situavam-se no interior de hospitais privados (três terceirizados e seis próprios); 19 no interior de hospitais públicos (todos próprios) e oito eram extra-hospitalares.

Em 2008, o LAC/DEFES/SESDEC inspecionou 119 LCs; desse número (Figura 3), 35\% não possuíam contrato com provedor de EP/CEQ. Desses 35\%, 23 unidades de LC estavam situadas no interior de hospitais privados (13 terceirizados e 10 próprios); 13 no interior de hospitais públicos (um terceirizado e 12 próprios) e seis eram extrahospitalares.

Dos LCs inspecionados em 2007 e 2008, 134 possuíam CEQ, dos quais 65\% não registram as ações corretivas relacionadas com as não conformidades detectadas em relatório emitido pelo provedor de EP, desacatando o item 7.2.4.1 da Portaria SES/CVS no 743, de 28 de junho de 2006, e o item 9.3.4 da RDC 302/2005.

Comparativamente, observa-se que o percentual de LCs privados, intra e extra-hospitalares, que cumpre o quesito normativo relativo ao CEQ é maior que o dos públicos e que, dentro da categoria LCs intra-hospitalares públicos, os municipais são os que cumprem em menor percentual esse quesito. Entretanto, ao longo do período analisado, houve aumento percentual dos $\mathrm{LCs}$ situados no interior de hospitais públicos que passaram a possuir CEQ: de $26 \%$ para $54 \%$.

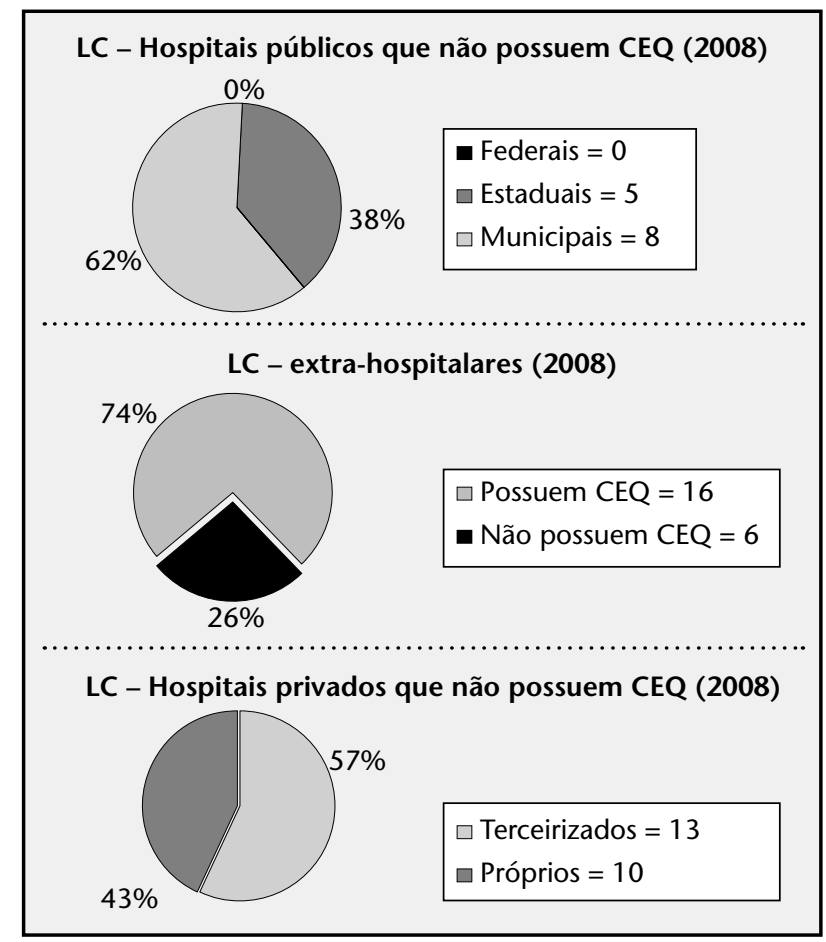

Figura 3 - Total de LCs (ano 2008) que não possuíam CEQ = 42 LC: laboratório clínico; CEQ: controle externo de qualidade. 


\section{Discussão}

Embora tenha sido observado que o número de LCs que respeitava a legislação sanitária vigente, quanto à implementação do CEQ, aumentou progressivamente na maioria dos casos após a publicação da RDC 302/2005, foi constado que um alto percentual de LCs não promovia ações corretivas quando a proficiência não era obtida, desacatando o item 7.2.4.1 da Portaria SES/CVS no-743, de 28 de junho de 2006, e o item 9.3.4 da RDC 302/2005. De acordo com a análise de dados feita para os anos de 2007 e 2008, relativas à promoção de ações corretivas para inadequações de algum analito do CEQ, o percentual observado é aproximadamente igual para os dois anos pesquisados.

Todavia, como a comprovação inicial de que o LC cumpre com a lei ocorre por meio do contrato com provedores de EP, os laboratórios apresentam este contrato, inclusive possuem o último relatório emitido pelo provedor, entretanto não apresentam registros das medidas corretivas pertinentes em que a proficiência não foi obtida em $65 \%$ dos 134 laboratórios inspecionados no âmbito do estado do Rio de Janeiro no período de 2007-2008 (o ano de 2006 não foi contabilizado, pois a Portaria 743/2006 só foi publicada na metade do ano de 2006), demonstrando que, efetivamente, não haverá contribuição para a melhoria da qualidade dos serviços laboratoriais prestados nem garantia da confiabilidade dos resultados expressos nos laudos emitidos.

\section{Conclusão}

Os LCs do estado do Rio de Janeiro, após a publicação da RDC 302/2005 pela ANVISA, vêm apresentado melhoria relativa ao cumprimento da legislação vigente, relacionada com o CEQ.

Podemos afirmar que a RDC 302/2005 é um avanço na regulamentação de LCs no Brasil, contudo o esforço para a adequação à legislação vigente por parte dos laboratórios clínicos do país está apenas começando. Os LCs deverão implantar o Programa de Ensaios de Proficiência na íntegra e buscar estratégias de gestão da qualidade, aplicando ações corretivas para as não conformidades observadas, visando a garantia da qualidade das análises laboratoriais, o diagnóstico preciso e a minimização dos impactos negativos sobre a saúde dos pacientes.

\section{Referências}

1. ANVISA - AGÊNCIA NACIONAL DE VIGILÂNCIA SANITÁRIA. Resolução RDC $n^{\circ}$ 302, de 13 de outubro de 2005, que dispõe sobre o regulamento técnico para o funcionamento de Laboratórios Clínicos. Diário Oficial [da] República Federativa do Brasil. Brasília, 14 de outubro de 2005.

2. BRASIL. Congresso Nacional. Lei $8.080,19$ de setembro de 1990. Dispõe sobre as condições para a promoção, proteção e recuperação da saúde, a organização e o funcionamento dos serviços correspondentes e dá outras providências. Diário Oficial da República Federativa do Brasil. Brasília, 20 de setembro de 1990.
3. RIO DE JANEIRO (ESTADO). Portaria SES/CVS no 743 , de 28 de junho de 2006. Institui os roteiros para a inspeção em laboratórios de análises clínicas, postos de coleta e serviços de execução de testes laboratoriais remotos (TLR) e testes rápidos em hospitais no âmbito do Estado do Rio de Janeiro e dá outras providências. Diário Oficial do Estado do Rio de Janeiro, Rio de Janeiro.

4. SCIACOVELLI, L., PLEBANI, M. The IFCC working group on laboratory errors and patient safety. Clinica Chimica Acta, v. 404, p. 79-85, 2009.

5. THOMAS, A. External Quality Assessment in laboratory medicine: is there a rationale to determine frequency of surveys? Accred Qual Assur, v. 14, p. 439-44, 2009. 